Ann. Geophysicae 16, 821-827 (1998) (C) EGS - Springer-Verlag 1998

\title{
Incoherent scatter radar observations of AGW/TID events generated by the moving solar terminator
}

\author{
V. G. Galushko ${ }^{1}$, V. V. Paznukhov ${ }^{1}$, Y. M. Yampolski ${ }^{1}$, and J. C. Foster ${ }^{2}$ \\ ${ }^{1}$ Institute of Radio Astronomy, National Academy of Sciences of Ukraine, Kharkov, Ukraine \\ ${ }^{2}$ MIT Haystack Observatory, Atmospheric Sciences Group, Westford, MA 01886, USA
}

Received: 13 October 1997 / Revised: 6 February 1998 / Accepted: 10 February 1998.

\begin{abstract}
Observations of traveling ionospheric disturbances (TIDs) associated with atmospheric gravity waves (AGWs) generated by the moving solar terminator have been made with the Millstone Hill incoherent scatter radar. Three experiments near 1995 fall equinox measured the AGW/TID velocity and direction of motion. Spectral and cross-correlation analysis of the ionospheric density observations indicates that STgenerated AGWs/TIDs were observed during each experiment, with the more-pronounced effect occurring at sunrise. The strongest oscillations in the ionospheric parameters have periods of 1.5 to 2 hours. The group and phase velocities have been determined and show that the disturbances propagate in the horizontal plane perpendicular to the terminator with the group velocity of $300-400 \mathrm{~m} \mathrm{~s}^{-1}$ that corresponds to the ST speed at ionospheric heights. The high horizontal group velocity seems to contradict the accepted theory of AGW/TID propagation and indicates a need for additional investigation.
\end{abstract}

Key words. Ionosphere (wave propagation) · Meteorology and atmospheric dynamics (waves and tides)

\section{Introduction}

Investigations of the atmospheric gravity waves (AGW) manifesting themselves at ionospheric heights as traveling ionospheric disturbances (TID) are of great importance due to their role in the energy and momentum exchange between different regions of the upper atmo-

Correspondence to: J. C. Foster sphere. A distinctive characteristic of this kind of wavelike disturbances is their persistence (the main AGW parameters such as spatial periodicity, velocity and direction of motion are retained over great distances from their origin). Hines (1960) was the first to give a fundamental explanation of AGWs and their relationship to TIDs. Since then, a number of review papers (Yeh and Liu, 1972; Hines, 1974; Francis, 1975; Hines, 1980; Hunsucker, 1982; Mayr et al., 1990; Crowley, 1991; Leitinger, 1992; Hocke and Schlegel, 1996) have described the essential results of AGW/TID research. Gravity waves in the upper atmosphere can be observed either directly as neutral gas fluctuations (for example, with the use of interferometers or satellite-borne mass spectrometers) or indirectly as ionospheric plasma variations using various remote radio techniques (incoherent scatter, IS, HF Doppler, TEC, etc.). But despite the extensive research that has been performed, there are still many open questions in the problem of the generation and propagation of AGWs/TIDs.

AGWs can be generated by different sources either of natural or anthropogenic origin (particle precipitation at high latitudes, moving solar terminator, jet streams, wind shears, earthquakes, hurricanes, tsunami or industrial accidents, ionosphere modification experiments, powerful blasts, chemical releases, etc.). It is worth noting that among all the sources of gravity waves, the moving solar terminator (ST) has a special status since it is a predictable phenomenon whose characteristics are well known. The ST is characterized by sharp changes in atmospheric parameters such as energy, temperature, pressure, and electron density at a given altitude. The main energy source, the solar heat input and its absorption, depends on the photochemistry of the atmosphere together with convection and diffusion effects. In the vicinity of the ST, the atmospheric gas is not in an equilibrium state. This situation and the motion of the ST cause both waves and turbulence. Such processes have been reviewed by Somsikov and Ganguly (1995). Considering the ST as a stable and repetitive source of AGWs, one can derive information 
about atmospheric conditions from the response of the medium to this input. Such a source is suitable for investigating gravity wave parameters and could be used as an atmospheric diagnostic, using the ST-generated waves as probe signals. However, experimental measurements of the effects of the solar terminator as a generation region for AGWs are quite rare (Bezrodny et al., 1976; Popov and Yampolsky, 1981; Galushko and Yampolski, 1983; Beley et al., 1995). The work reported here was directed towards determining the main parameters of ST-generated AGWs/TIDs through a set of specifically designed and analyzed incoherent scatter radar observations.

\section{Experiments}

Experimental observations were obtained with the highpowered UHF $(440 \mathrm{MHz})$ incoherent scatter radar at the Millstone Hill Observatory $\left(42.6^{\circ} \mathrm{N}, 288.5^{\circ} \mathrm{E}\right)$. Ionospheric data investigated include electron density, electron temperature, ion temperature, and ion drift velocity observed along the line of sight of the fully steerable 46-meter or the zenith-directed 68-m antenna. An initial investigation sought direct evidence in the radar data of an ionospheric response to the passage of the solar terminator used previously-acquired data from a March 1992 zenith-directed, fixed-beam, radar experiment. Spectral analysis identified low-frequency disturbances with the period of more than $1 \mathrm{~h}$ which were generated by the ST. Subsequently, special experiments were carried out during the autumn of 1995 to investigate further these wavelike disturbances, and to determine their main parameters such as spatial periodicity, velocity, and direction of motion. These dedicated experiments were performed in near-equinox conditions when the terminator is best aligned along the meridian and were designed to look for the altitude/ latitude signatures, predicted by the ST/AGW-generation theory. To investigate altitude and space/time effects, a three-beam operating mode was chosen, whose scheme is shown in Fig. 1. Switching between antenna positions in the sequence "zenith-west-zenith-northzenith" was done with a cycle time of $5 \mathrm{~min}$ and data were integrated in each position for $68 \mathrm{~s}$. Hence, the sampling time was equal to $5 \mathrm{~min}$ for positions "west" and "north" and $2.5 \mathrm{~min}$ for the zenith position. Ionospheric parameters were determined over the height range $154 \mathrm{~km}-427 \mathrm{~km}$ with a height resolution of $\sim 20 \mathrm{~km}$.

In order to determine TID characteristics unambiguously, we need to have $\Delta t<T / 2$, where $\Delta t$ is the data sampling time and $T$ is the period of the TID. Spatial sampling is done with $\Delta L_{Z-W}^{h}, \Delta L_{Z-N}^{h}<\Lambda$, where $\Delta L_{Z-W}^{h}, \Delta L_{Z-N}^{h}$ are the horizontal spatial distances between positions "zenith-west" and "zenith-north" at height, $h$, and $\Lambda$ is the TID wavelength. As follows from theoretical works (e.g. Somsikov, 1983; Somsikov and Ganguly, 1995) the temporal period and spatial scale of ST-generated TIDs depend on the Mach number $M=V_{T} / V_{S}$, where $V_{T}$ is the velocity of the $\mathrm{ST}$ and $V_{S}$

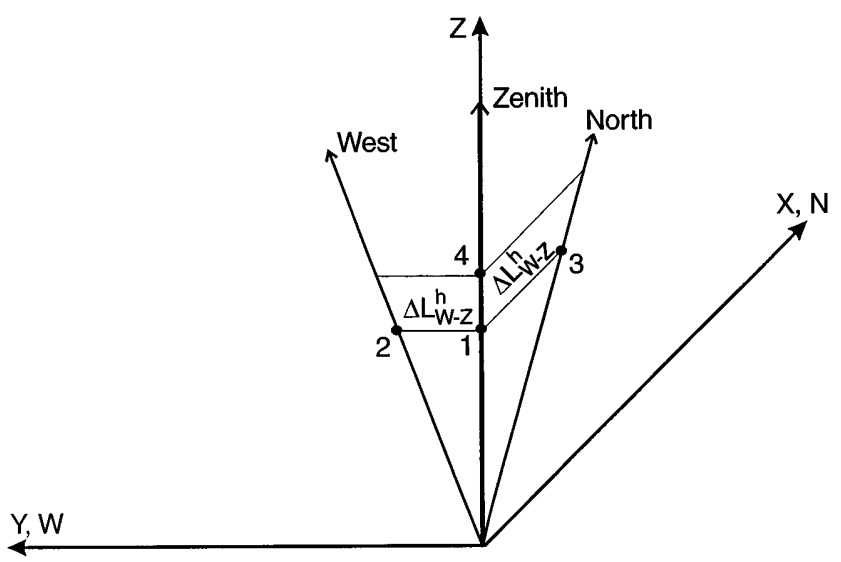

Fig. 1. Schematic representation of the 3-beam radar experiment used to determine TID/AGW propagation characteristics. Radar beams at $40^{\circ}$ elevation angle to the north and the west were interleaved with vertical observations with the Millstone Hill zenith antenna

is the sound velocity. At mid-latitudes, three regions of the atmosphere can be differentiated based on the magnitude of $M$. The first corresponds to inequality $M^{2}>1$ and is located below the altitude of $120 \mathrm{~km}$. Both acoustic and gravity waves can be generated in this region. The second region lies between $120 \mathrm{~km}$ and $180 \mathrm{~km}$ where $1>M^{2}>0.8$. The waves generated by the ST in this region, as a rule, are decaying. Finally, a third region where $M^{2}<0.8$ begins from $\sim 180 \mathrm{~km}$ and stretches up to $600 \mathrm{~km}$. In this region the ST generates gravity waves with a spatial scale of about $1000 \mathrm{~km}$ and with period more than $30 \mathrm{~min}$. Taking into account the location of the Millstone Hill Observatory and the height range where the measurements of the ionospheric parameters were performed, one concludes that these experiments are most likely to observe TIDs related with gravity waves with spatial scales of a few hundreds of kilometers.

It is known (Hargreaves, 1982) that gravity waves have frequencies less than Brunt-Väisälä frequency, $\omega_{B}$. For the height range where the measurements were carried out, the period corresponding to $\omega_{B}$ is $T_{B}=1 / \omega_{B} \approx 10 \mathrm{~min}$. Therefore, the shortest expected TID period is equal to $10 \mathrm{~min}$ and an experimental sampling time of 5 min was chosen. For a rough estimate of TID wavelength, we assume that the disturbances move at the velocity of the ST, which varies slightly over the height range of interest. For $240 \mathrm{~km}$ (near the height of the daytime electron density maximum), the TID period was assumed to be equal to the shortest period expected, $10 \mathrm{~min}$. Thus, the shortest expected wavelength is $\Lambda=V_{T} \times T=360 \mathrm{~km} \mathrm{~s}^{-1} \times$ $600 \mathrm{~s}=216 \mathrm{~km}$.

So, it is necessary that $\Delta L_{Z-W}^{240}$ and $\Delta L_{Z-N}^{240}$ be less than $216 \mathrm{~km}$. In our measurements they both were chosen to equal $200 \mathrm{~km}$ by setting the elevation angle of antenna beams to be $40^{\circ}$ from vertical. As mentioned already, the most likely TID periods are expected to be greater than $30 \mathrm{~min}$, and therefore the experiment plan was well matched to its objectives. 


\section{Data processing}

Of the four ionospheric parameters investigated, the STgenerated disturbances were most consistently observed in the electron density data. Segments of electron density records obtained on September 21, 1995 are shown in Fig. 2. Curves for different heights are artificially shifted to avoid overlapping. Times of the sunset and sunrise terminator passage at ionospheric heights over the observatory are indicated. The ST-generated disturbances manifest themselves as a "peak" in electron density at sunset as well as "fast" oscillations after sunrise. However, the pronounced diurnal trend of the parameters makes it difficult to observe the effect of AGWs/TIDs generated by the ST and complicates their spectral analysis. Accordingly, the data were detrended
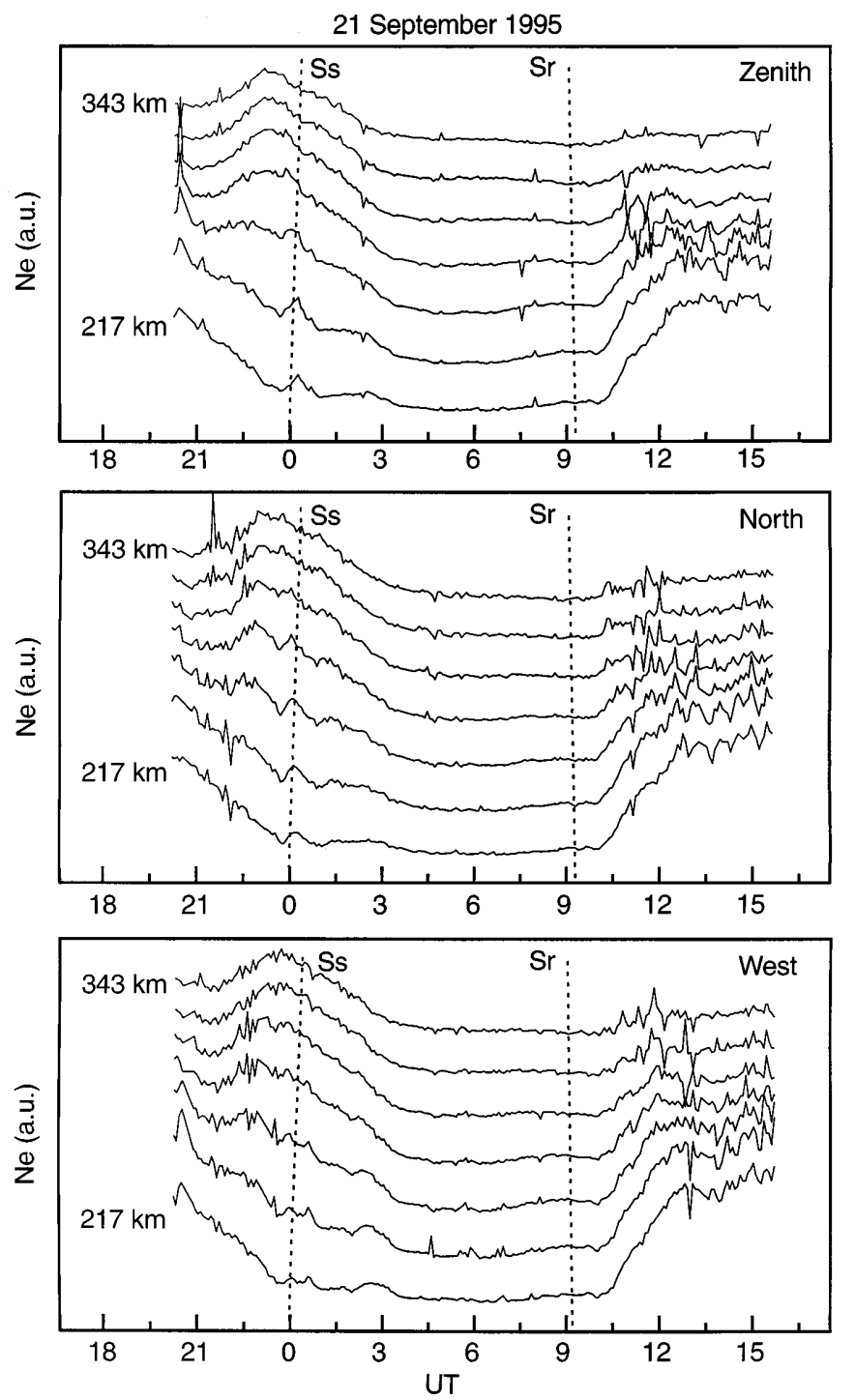

Fig. 2. Unfiltered electron density observations (log 10$)$ observed at several altitudes during the dedicated TID experiment on September 21, 1995 are shown for the three antenna positions of Fig. 1. Curves for different heights are artificially shifted to avoid overlapping. Times of the sunset and sunrise terminator passage at ionospheric heights over the observatory are indicated before further processing by subtracting data smoothed by a moving rectangular time window. Disturbances with periods $>2.5 \mathrm{~h}-3 \mathrm{~h}$ can be associated with tidal modes (Hocke, 1996), so a smoothing window width of $2.5 \mathrm{~h}$ was chosen to remove variations which were not associated with the ST. This slowly varying trend, $\langle N\rangle$, was subtracted from the original density data to yield the time-varying part of the electron density record, $\delta N=N-\langle N\rangle$, which was further analyzed with a stepped Fourier transform

$S\left(\omega, T_{s}+n \cdot \Delta t\right)=\int_{T_{s}+n \cdot \Delta t}^{T_{s}+n \cdot \Delta t+T_{j}} \mathrm{~d} t \delta N e^{-j \omega t}$

which determines a time-sequence of spectra where $\Delta t$ is the step between adjacent spectra, $n=1,2,3, \ldots, T_{j}$ is the integration time, $\omega=2 \pi f$ is the angular frequency, $T_{s}$ corresponds to the start of the records. The results of applying this procedure to the data collected in the zenith position on September 21, 1995 are presented in Fig. 3 where $T_{j}$ was $2.5 \mathrm{~h}$, and $\Delta t$ was $10 \mathrm{~min}$ (2 samples). Some strengthening of the spectral intensity after sunrise and sunset over a wide height range is apparent. It is worth noting that the strengthening of the fluctuations does not occur across the entire frequency range, but only at selected frequencies (this is quite evident for the spectra at $301 \mathrm{~km}$ ). Such spectral fine structure indicates the presence of wavelike processes in the ionosphere. From Fig. 3 one can see that the most powerful spectral components are concentrated within the frequency band which corresponds to time periods of 1.5-2.5 h. The most powerful spectral components then were selected by a numerical rectangular filter. The band-pass filter had cutoff frequencies corresponding to the time periods $T_{\min }=90 \mathrm{~min}$ and $T_{\max }=130 \mathrm{~min}$. Figure 4 demonstrates filtered data for the three antenna positions ("zenith", "west", and "north"). The spatial/temporal structure of the disturbance is represented by the isolines of electron density and the intensity of the fluctuations is given in gray scale. The maximum intensity of the disturbances is observed near the solar terminator. It is clearly seen that the effect is not over the whole height range, but occurs only at heights lower than $\sim 300 \mathrm{~km}$.

We have used a triangular method to determine TID characteristics, including velocity and direction of motion. To estimate these parameters, measurements are needed in four different points, not in the same plane (Fig. 1, points 1-4). To determine time delays between variations for different measuring points, cross-correlation analysis was applied to the filtered records near the time of ST passage for positions "zenith", "west", and "north" and results are given in Table 1. The crosscorrelation values are sufficiently high over the height range $217-301 \mathrm{~km}$ to conclude that the disturbances are moving as "frozen-in irregularities", i.e., all spectral components move with the same velocity. The time delays between variations for the different points were derived from the maxima of the correlation functions. TID group velocity, $V_{g}$, and direction of motion were 


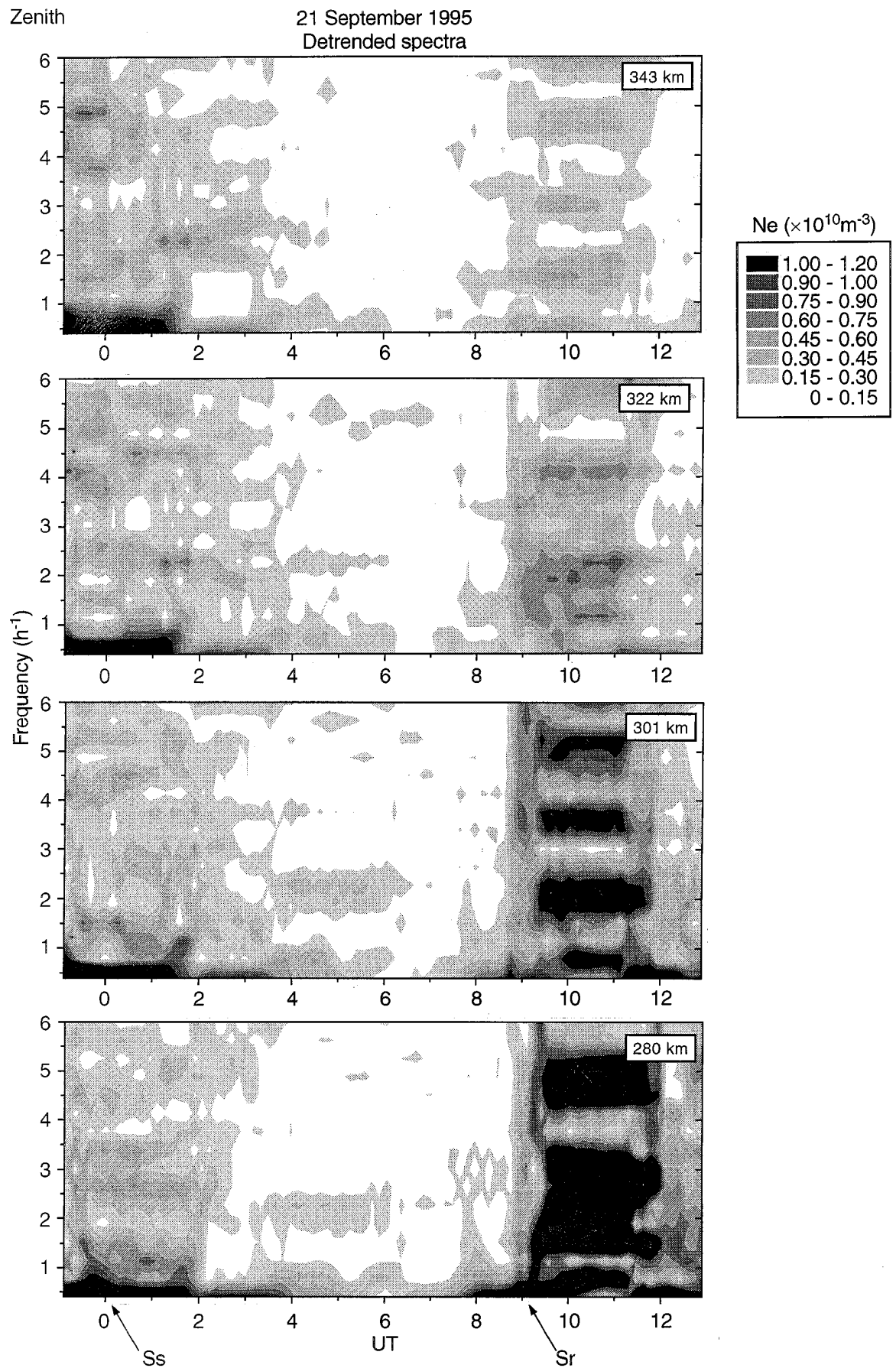

Fig. 3. Spectral power of the detrended density data at four $F$-region altitudes reveal strong intensification of wavelike (banded) disturbances in the two hours following sunrise (at $\sim 09$ UT). The most powerful spectral components correspond to temporal periods of $1.5 \mathrm{~h}-2.5 \mathrm{~h}$ determined for the height range where values of crosscorrelation functions were high. The results of these estimates of $V_{g}$ are given in Table 2. In the frame of reference where $V_{g}$ was derived, $\gamma$ is elevation angle and $\phi$ is azimuth measured counter-clockwise from the north. TID phase velocity was determined as the velocity of a monochromatic wave. To estimate the phase velocity of the TID motion, the strongest spectral component ( $2 h$-wave) was selected from the spectrum of the filtered records. Phase differences between variations for four measuring points were derived from the crossspectra. Propagation velocity of the $2 h$-wave was estimated as the speed of motion of a plane wave. The phase velocity and direction of propagation of the strongest component of the wave packet are given in Table 3 .

We are able to determine the spatial wavelength of the disturbances from the temporal period and velocity of the TIDs motion. Figure 5 illustrates $V_{p h}$ versus $\Lambda$ dependencies which follow the dispersion law for ionospheric wavelike processes. The data were derived for the most powerful spectral harmonics of the electron density variations for different heights. It is evident that for longer wavelengths, the wave moves faster. A similar behavior of AGWs has been mentioned by other authors (e.g. Hocke and Schlegel, 1996). 

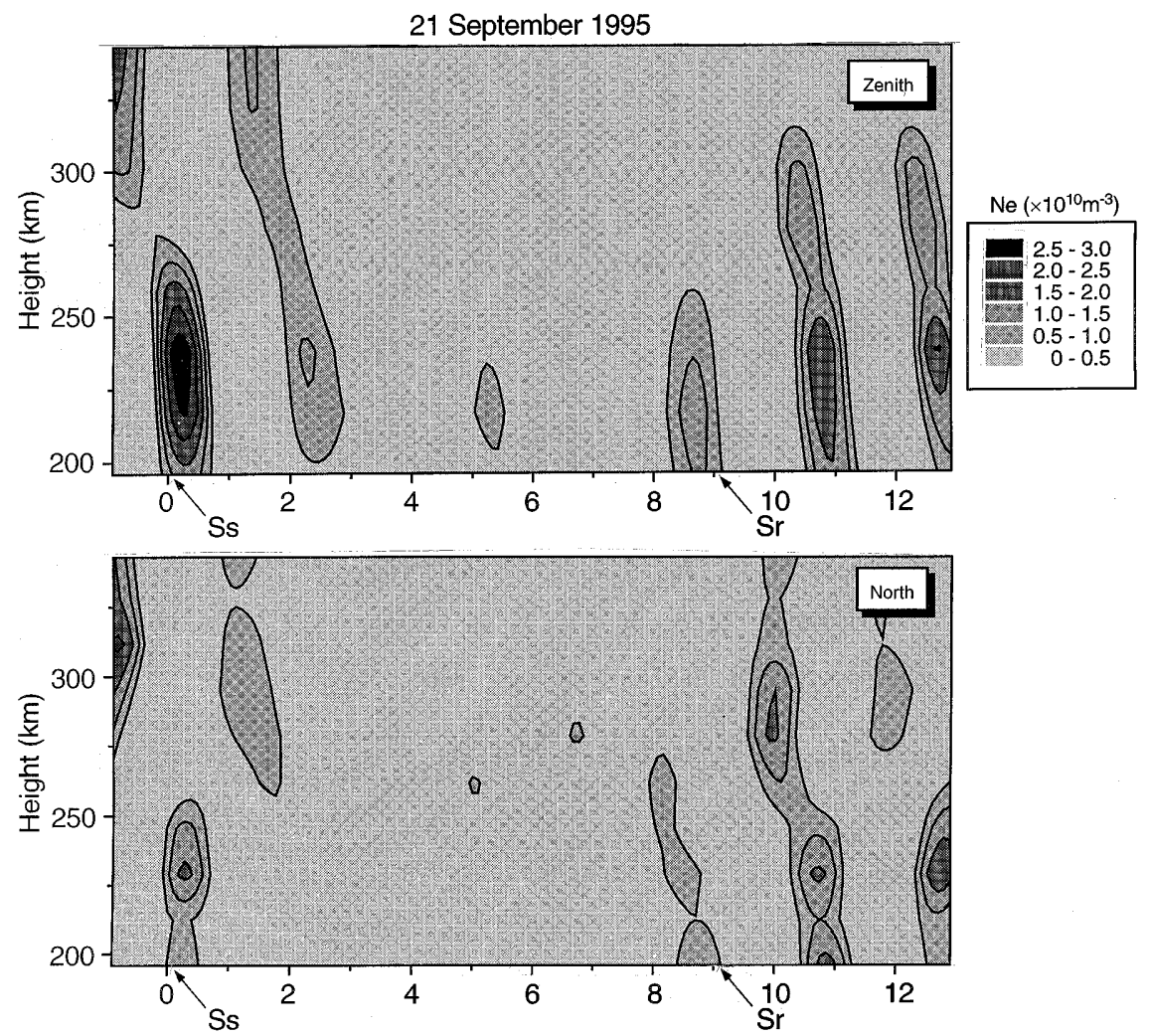

Fig. 4. Intensity of the disturbance in the filtered spectral band $90 \mathrm{~min}-130 \mathrm{~min}$ for the three observing positions and across $F$-region altitudes indicates intensification of the disturbances at altitudes $<300 \mathrm{~km}$ at the times

of sunset and sunrise terminator passage

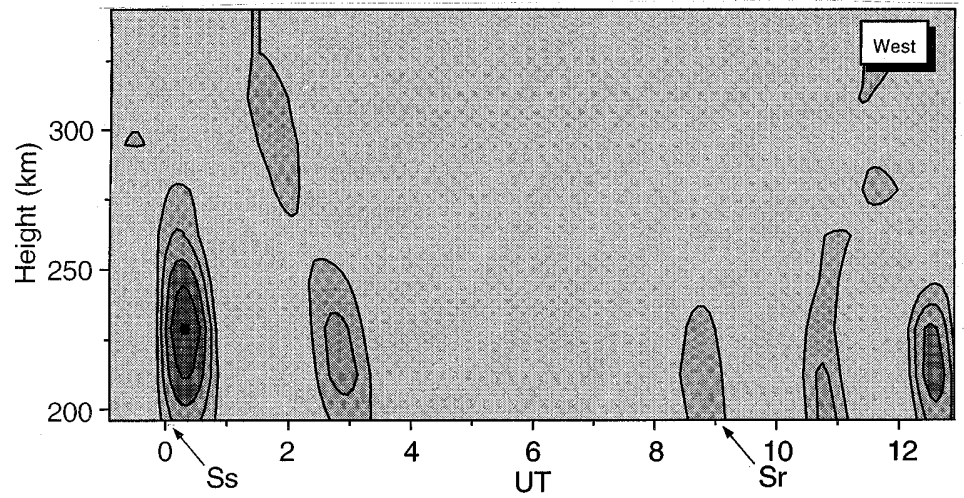

\section{Discussion}

The results of our investigations demonstrate that TIDs are generated by the moving solar terminator in the ionosphere. As seen in Fig. 3, the effect is observed best at sunrise, while at sunset it is rare. The measured velocity and direction of the propagation strongly

Table 1. Cross-correlation coefficient for positions $\mathrm{Z}, \mathrm{W}$, and $\mathrm{N}$ near the time of ST passage

\begin{tabular}{lll}
\hline$h, \mathrm{~km}$ & $\mathrm{~W}-\mathrm{Z}$ & $\mathrm{N}-\mathrm{Z}$ \\
\hline 196 & 0.80 & 0.60 \\
217 & 0.83 & 0.75 \\
238 & 0.95 & 0.91 \\
259 & 0.90 & 0.87 \\
280 & 0.84 & 0.63 \\
301 & 0.62 & 0.45 \\
322 & 0.40 & 0.38 \\
\hline
\end{tabular}

Table 3. TID phase velocity and direction of propagation of the strongest (2-h) component

\begin{tabular}{llll}
\hline$h, \mathrm{~km}$ & $V_{p h}, \mathrm{~m} / \mathrm{s}$, & $\gamma, \operatorname{deg}$ & $\phi, \operatorname{deg}$ \\
\hline 217 & 550 & -3 & 17 \\
238 & 193 & 62 & 253 \\
259 & 268 & 51 & 212 \\
280 & 165 & 27 & -49 \\
\hline
\end{tabular}

Table 2. TID group velocity $\left(V_{g}\right)$ and direction of motion ( $\gamma$ elevation angle and $\phi$ azimuth)

\begin{tabular}{llll}
\hline$h, \mathrm{~km}$ & $V_{g}, \mathrm{~m} / \mathrm{s}$ & $\gamma, \mathrm{deg}$ & $\phi, \mathrm{deg}$ \\
\hline 217 & 475 & 0 & 101 \\
238 & 343 & 0 & 98 \\
259 & 268 & 0 & 96 \\
280 & 268 & 0 & 96 \\
301 & 236 & 0 & 120 \\
\hline
\end{tabular}



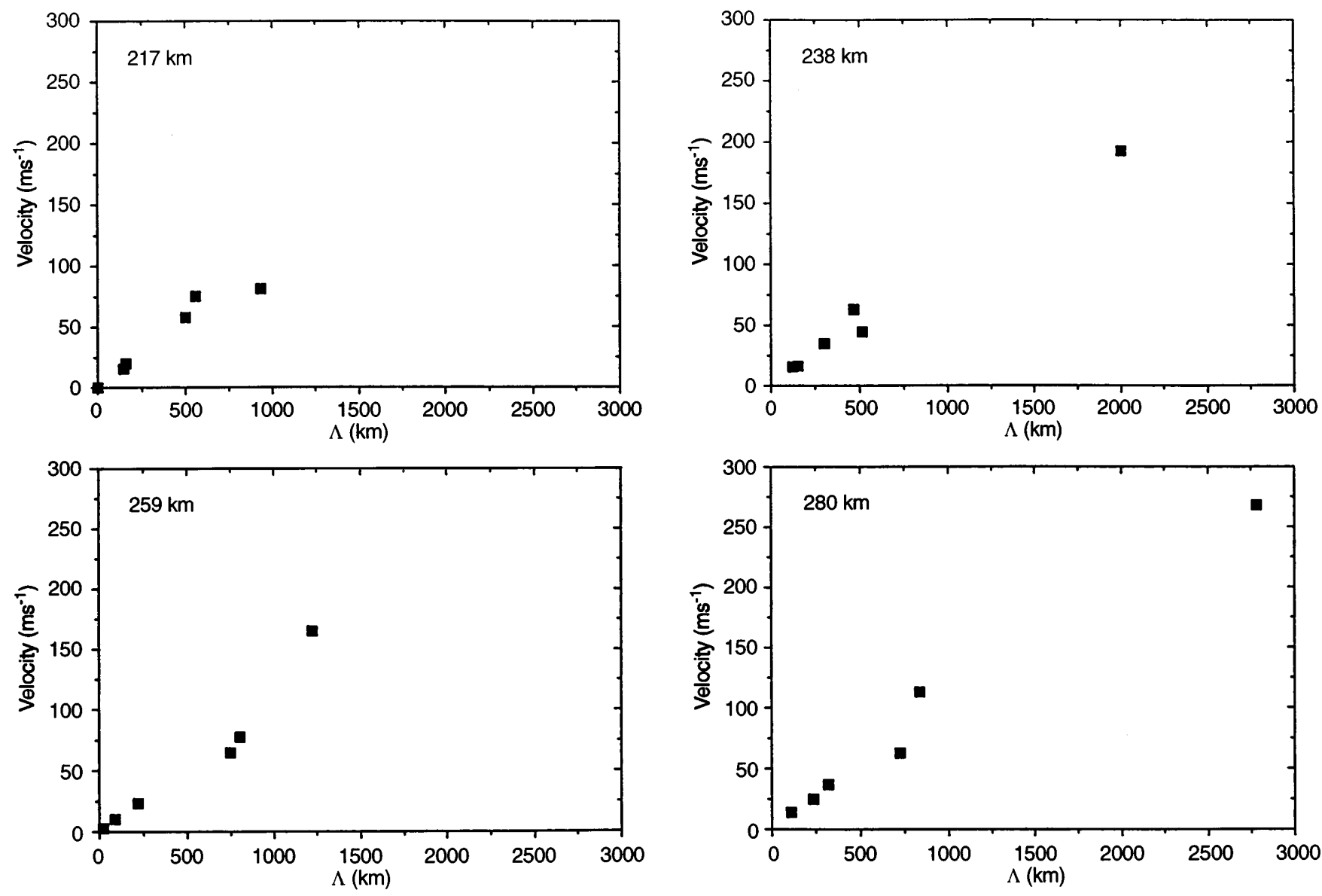

Fig. 5. $V_{p h}$ versus $\Lambda$ has been derived for the most powerful spectral components of the electron density variations for different heights. These follow the dispersion law for ionospheric wavelike processes and is evident that for longer wavelengths, the wave moves faster

indicate that the observed TIDs are associated with the ST. First, the group velocity, $V_{g}$, is close to the terminator velocity at ionospheric heights $\left(V_{t} \approx 360 \mathrm{~m} \mathrm{~s}^{-1}\right)$. Second, propagation is directed to the west (i.e., perpendicular to the terminator). Our results indicate that the ST generates low frequency disturbances of large spatial scale. Figure 5 shows that TIDs with spatial wavelengths from hundreds to a thousand kilometers are detected most frequently. This implies that the observed TIDs are connected with internal waves belonging to the gravity part of AGW. The observations support theoretical predictions of the expected spatial scale of STgenerated TIDs, in keeping with the model of ST influence on the ionosphere described in Somsikov (1983).

It is known (Williams, 1996) that under atmospheric conditions AGW phase velocity and group velocity are perpendicular to each other. The angle between $V_{g}$ and $V_{p h}$ obtained in these experiments may be derived through a comparison of Table 2 and Table 3, whose results are given in Table 4. It can be seen that over the height range $217-259 \mathrm{~km}$, the angle between $V_{g}$ and $V_{p h}$

Table 4. Angle between TID group and phase velocities

\begin{tabular}{lrlll}
\hline$h, \mathrm{~km}$ & 217 & 238 & 259 & 280 \\
$\Delta \phi, \mathrm{deg}$ & 84 & 115 & 105 & 137 \\
\hline
\end{tabular}

is close to $90^{\circ}$. This fact indicates that the observed disturbances are of AGW origin.

An interesting result concerns the observation of horizontal motion for the TIDs (Table 2). In accordance with the accepted theory, only a wave which has a frequency close to the Brunt-Väisälä frequency is able to propagate horizontally in the atmosphere. Following Hargreaves (1979), the angle of elevation for AGW propagation can be derived as

$\gamma=\operatorname{atan}\left(\omega_{B}^{2} / \omega^{2}-1\right)^{1 / 2}$

where $\omega$ is the frequency of the AGW, $\omega_{B}$ is the BruntVäisälä frequency. Consequently, if $\omega \ll \omega_{B}$ (which is the case) then $\gamma \approx 90^{\circ}$ and the wave under consideration should propagate vertically. This contradiction probably arises from the fact that expression (2) applies to AGWs (i.e., to neutral gas variations), while charged component variations (TIDs) were measured in the experiments. The relationship between TIDs and AGWs is complicated by the necessity of taking into account the influence of the Earth's magnetic field on the motion of electron density structure in the ionosphere.

AGW parameters are closely related to the characteristics of the atmosphere. For instance, the velocity of sound in the ionosphere can be found from the ratio of phase to group velocity observed in such experiments. A new method for upper atmosphere/ionosphere diagnos- 
tics using wavelike disturbances generated by the moving solar terminator is suggested, based on the interpretation of such results.

\section{Conclusions}

The generation of ionospheric disturbances by the moving solar terminator (ST) has been confirmed with the aid of the three-position incoherent scatter radar experiments and the use of special data-processing algorithms. It was shown that the strongest components of TIDs associated with the ST have temporal periods of about $2 \mathrm{~h}$. The TIDs were shown to move horizontally, perpendicular to the terminator, and the occurrence of dominant horizontal motion seems to contradict the accepted theory of AGW/TID propagation, indicating a need for additional investigations, both theoretical and experimental. The use of ST-generated disturbances as probe signals is suggested as a new technique for atmospheric diagnostics.

Acknowledgements. The authors wish to thank the colleagues of the Millstone Hill Observatory who have taken part in these experiments. Millstone Hill radar observations are supported by the US National Science Foundation through cooperative agreement with the Massachusetts Institute of Technology.

Topical Editor D. Alcaydé thanks R. D. Hunsucker and K. Hocke for their help in evaluating this paper.

\section{References}

Beley V. S., V. G. Galushko, and Y. M. Yampolski, Traveling ionospheric disturbances diagnostic using HF signal trajectory parameter variations, Radio Sci. 30(6), 1735-1752, 1995.

Bezrodny Y. G., Y. M. Yampolski, On the origin of the spatial phase deference of the VLF at sunrise and sunset period, Izvestia VUZ-Radiofizika (Soviet Radiophysics and Quantum electronics), 19(9) , 1270-1274, 1976.
Crowley, G., Dynamics of the Earth's thermosphere; a review, Rev. Geophys., 29, 1143-1165, 1991.

Francis S. H., Global propagation of atmospheric gravity wave; a review, J. Atmos. Terr. Phys., 37, 1011-1054, 1975.

Galushko V. G., Y. M. Yampolski, Experimental investigations of the HF signals scattered by moving solar terminator, Izv. VUZRadiofiz., 26(4), 499-502, 1983.

Hargreaves, J. K., The upper atmosphere and solar-terrestrial relations. An introduction to the aerospace environment, Van Nostrand Reinhold, 1982.

Hines, C. O., Internal atmospheric gravity waves at ionospheric heights, Can. J. Phys., 38, 1441-1481, 1960.

Hines, C. O., The upper atmosphere in motion, Geophys. Monogr. Ser. 18, AGU, Washington D. C., 1974.

Hines, C. O., Earlier days of gravity waves revisited, Pure Appl. Geophys., 130, 151-170, 1980.

Hocke, K., Tidal variations in the high-latitude E-region observed by EISCAT, Ann. Geophysicae, 14, 201-210, 1996.

Hocke K., and K. Schlegel, A review of atmospheric gravity waves and travelling ionospheric disturbances: 1982-1995, Ann. Geophysicae, 14, 917-940, 1996.

Hunsucker, R., Atmospheric gravity waves generated in highlatitude ionosphere: a review, Rev. Geophys. Space Phys., 20, 293-315, 1982.

Leitinger, R., Travelling ionospheric disturbances (TIDs) - Wissensstand und neuere Entwicklungen, Kleinheubacher Ber., 35, 1-14, Deutsche Telekom, 1992.

Mayr, H. G., I. Harris, F. Varosi, and F. A. Herrero, Thermospheric gravity waves: observation and interpretation using transfer function model (TMF), Space Sci. Rev., 54, 297-375, 1990.

Popov A. V., Y.M. Yampolski, VLF variations during sunrise period, Izve. VUZ-Radiofiz., 24(6), 1981, 794-798.

Somsikov, V. M., Solar terminator and dynamics of the atmosphere, Nauka, Alma-Ata, 1983.

Somsikov, V. M., and B. Ganguly, On the mechanism of formation of atmospheric inhomogeneties in the solar terminator region, J. Atmos. Terr. Phys., 57, 75-83, 1995.

Williams P. J. S., Tides, atmospheric gravity waves and travelling disturbances in the ionosphere. Modern ionospheric science. European Geophysical Society, Katlenburg-Lindau, FRG 1996.

Yeh, K. C., and C. H. Liu, Theory of ionospheric waves, Academic Press, New York-London 